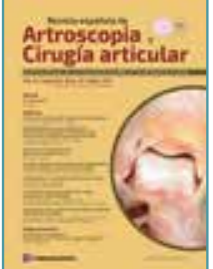

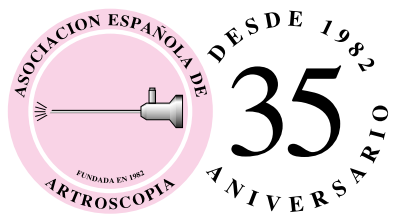

Vol. 24. Especial. Núm. 57. Mayo 2017

ISSN: 2386-3129 (impreso)

2443-9754 (online)

\title{
Original
}

\section{Indicaciones de la artrolisis artroscópica en rigidez tras prótesis de rodilla}

\author{
F. Maculé', P. Hinarejos², J. Leal'², E. Castellet ${ }^{3}$, O. Ares' ${ }^{1}$, P. Barrena ${ }^{4}$, \\ M. aL. Bertrand ${ }^{5}$, V.J. León ${ }^{6}$, E. Montañez ${ }^{7}$, F.J. Montilla ${ }^{8}$ \\ ${ }^{1}$ Hospital Clínic. Barcelona; 2 Parc de Salut Mar. Barcelona; ${ }^{3}$ Hospital Vall d'Hebron. Barcelona \\ ${ }^{4}$ Hospital Universitario Virgen del Rocío. Sevilla; ${ }^{5}$ Hospital Costa del Sol. Marbella, Málaga \\ ${ }^{6}$ Hospital de la Vega Lorenzo Guirao. Cieza, Murcia; ${ }^{7}$ Hospital Universitario Virgen de la Victoria. Málaga \\ ${ }^{8}$ Hospital Viamed Santa Ángela de la Cruz. Sevilla
}

Correspondencia:

Dr. Francisco Maculé

Correo electrónico: fmacule@gmail.com
Recibido el 28 de marzo de 2017

Aceptado el 5 de abril de 2017

Disponible en Internet: mayo 2017

\section{RESUMEN}

La rigidez de rodilla tras una artroplastia es una alteración grave que provoca dolor y condiciona el futuro de la articulación. Las causas pueden ser primarias, cuando modifican la anatomía articular (genética, artrosis, deformidades, etc.) o secundarias, si están situadas fuera de la articulación. Dentro de estas, pueden ser atribuibles al paciente (por ejemplo, falta de movilidad previa) y/o a errores técnicos (asimetría de espacios, osteofitos posteriores, implante femoral en rotación interna, mal balance de ligamentos, sobredimensionamiento de los implantes, etc.) y complicaciones (por ejemplo, infección, fracturas pre- y periprotésicas, distrofias, etc.). Para un correcto tratamiento se debe identificar la etiología y solo en caso de no tener un diagnóstico de certeza, vamos a reconocer la artrofibrosis como causa de la rigidez, como un proceso de metaplasia del fibrocartílago con incremento de la fibrosis intersticial. En los casos de rigideces precoces, el tratamiento incluye una rehabilitación agresiva con una analgesia adecuada y suficiente. La técnica quirúrgica requiere un orden en el desbridamiento, que se inicia en el espacio intercondilar siguiendo por los recesos medial y lateral hasta llegar a la zona suprapatelar, donde reside la primera causa de limitación de la flexión. En caso de persistir la limitación de la extensión, se

\section{ABSTRACT \\ Indications of arthroscopic lysis in stiffness after knee prosthesis}

Stiffness after total knee replacement is a serious disorder that causes pain and determines the future of the joint. The causes may be primary, when they modified the anatomy (genetics, arthritis, deformities, etc.) or secondary, if they are located outside the joint. Some of them may be attributable to the patient (e.g., lack of prior mobility) or technical errors (asymmetry of gaps, osteophytes, femoral implant in internal rotation, soft tissue balancing, oversizing of implants, etc.) and complications (e.g., infection, fractures, dystrophies, etc.).

For proper treatment, we must identify the etiology. Only if there is no diagnosis of certainty, we will recognize the arthrofibrosis as a cause of the stiffness as a process of metaplasia of the fibrocartilage with an increase of interstitial fibrosis. In cases of early stiffness, the treatment includes aggressive rehabilitation with adequate analgesia. The surgical technique requires an order in the debridement, which starts in the intercondylar space following medial and lateral gutter to the suprapatelar pouch where the first cause of limitation of flexion resides. If the limitation

https://doi.org/10.24129/j.reaca.24e57.fs1704015

FS @ 2017 Fundación Española de Artroscopia. Publicado por Imaidea Interactiva en FONDOSCIENCE ${ }^{\circledR}$ (www.fondoscience.com). Este es un artículo Open Access bajo la licencia CC BY-NC-ND (www.creativecommons.org/licenses/by-nc-nd/4.0/). 
accede al compartimento posterior para liberar e incluso desinsertar la cápsula posterior.

Palabras clave: Artroscopia. Artroplastia total rodilla. Rigidez. Artrofibrosis. Artrolisis. of the extension persists, we access to posterior compartment to release and even mobilise the posterior capsule.

Key words: Arthroscopy. Total knee replacement. Stiffness. Arthrofibrosis. Arthrolysis.

\section{Introducción}

La limitación de la movilidad de la rodilla tras una artroplastia es una grave alteración que provoca dolor y condiciona la biomecánica articular desde la posición estática hasta la deambulación, siendo la principal causa de insatisfacción en la cirugía protésica de rodilla. Los déficits de la extensión activa provocan una marcha con cojera ostensible que se manifiesta en dolor en la cara anterior de la rodilla y que, a medio plazo, afectará a las articulaciones vecinas de cadera y tobillos, así como a la columna lumbar. La definición de rodilla rígida es muy variable y Aglietti et al.(1) la consideran con un arco de movimiento menor de $50^{\circ}$, pero Scranton ${ }^{(2)}$ considera rigidez una flexión menor de $85^{\circ}$, mientras Christensen et al. ${ }^{(3)}$, con menos de $75^{\circ}$ o una contractura en flexión de más de $15^{\circ}$ o un arco de movimiento menor de $45^{\circ}$ en cualquier momento del recorrido articular $^{(4,5)}$.

Entre las causas primarias de la rigidez articular se encuentran todas aquellas enfermedades que alteran la anatomía articular, como son la artrosis, la obesidad, la genética, las deformidades axiales, la espondilitis anquilopoyética, la artritis reumatoide juvenil o artritis o una infección articular posquirúrgica. Entre las causas secundarias asientan diferentes procesos patológicos que afectan a estructuras extraarticulares que pueden también alterar el arco de movimiento y crear confusión en cuanto a la causa principal o secundaria del origen de la rigidez $z^{(5,6)}$, la movilidad de la cadera ipsilateral y las lesiones neurológicas sistémicas.

El "síndrome doloroso regional complejo" tiene una prevalencia en cirugía de rodilla de hasta un $21 \%$ y es señalado como el causante de un elevado porcentaje de rodillas dolorosas posquirúrgicas sin causa aparente ${ }^{(7)}$. La lesión de la rama infrapatelar del nervio safeno despierta, en cada caso, en cada situación y de forma muy individual, diferentes sensaciones locales que van desde la simple hipoestesia bien tolerada hasta el dolor insoportable en la cara lateral y anterior de la articulación.

Existe una íntima relación entre dolor y rigidez, por lo que la solución de este problema debe ser orientado hacia la eliminación de los probables focos de dolor, la percepción de otros factores externos que influyan en el problema, el diagnóstico de posibles errores en la cirugía local y el tratamiento personalizado de cada situación. Las manipulaciones bajo anestesia en periodos precoces tras la realización de una artroplastia y con una falta de movilidad sin causa aparente pueden ser un excelente método como ayuda durante el periodo de rehabilitación $n^{(8)}$, aunque no está exento de problemas y probablemente tenga que acompañarse de técnicas artroscópicas con buenos resultados ${ }^{(9)}$.

Existen análisis de requerimientos de movilidad que muestran la movilidad mínima para determinadas actividades ${ }^{(10,11)}$. En fase oscilatoria de la marcha son $67^{\circ}$ de flexión, para subir y bajar escaleras $83^{\circ}$ y $93^{\circ}$ para levantarse de una butaca.

La prevalencia de esta complicación no está clara. Kim et al. ${ }^{(12)}$ refieren una tasa de 1,3\%, con un promedio de 32 meses después de la intervención, en un estudio de 1.000 prótesis de rodilla primarias consecutivas. En cambio, otros autores ${ }^{(13,14)}$ han establecido una tasa entre el 8 y el $12 \%$.

Dentro de las etiologías secundarias vamos a diferenciar los factores atribuibles a los pacientes y las que son debidas a errores técnicos de la intervención y complicaciones de la misma.

\section{Factores de los pacientes}

Una de las variables más importantes es la movilidad previa de la rodilla ${ }^{(5,13)}$; Kim et al. ${ }^{(12)}$ encuentran una disminución significativa de la movilidad en el preoperatorio en todos los pacientes que presentaron rigidez de rodilla tras la intervención. Pacientes obesos y con poca estatura también pre- 
sentan con mayor frecuencia una disminución del arco de movimiento por pinzamiento de las partes blandas de la zona posterior de la rodilla ${ }^{(5,13)}$. La tendencia a la formación de cicatrices queloides, así como desaxaciones preoperatorias, tanto en varo como en valgo, en pacientes jóvenes ${ }^{(14)}$ son factores que predisponen a una limitación de la flexión postoperatoria, incluso aunque la técnica quirúrgica sea perfecta ${ }^{(10)}$. La falta de colaboración con el programa de rehabilitación funcional y la poca tolerancia al dolor dificultan la obtención de unos parámetros aceptables de movilidad. Por último, pueden presentar una tendencia a la rigidez postoperatoria aquellos pacientes con problemas psicológicos, como depresiones importantes o abuso de psicofármacos o drogas, o trabajadores con conflicto de intereses económicos ${ }^{(10)}$.

\section{Errores técnicos y complicaciones}

Probablemente, la mayoría de las rodillas rígidas después de una artroplastia son debidas a errores técnicos durante la intervención ${ }^{(10)}$ y una causa frecuente de limitación del arco de movilidad postoperatoria es por no liberar la cápsula posterior retraída, no extraer los osteofitos posteriores $^{(5,13)}$ o tener los espacios en flexión y extensión mal equilibrados.

Ante la realización de un corte distal femoral insuficiente, con la consiguiente disminución del espacio en extensión, probablemente aparecerá una limitación de la extensión con una rótula baja (Figura 1). Una altura inadecuada de la interlínea articular produce un desequilibrio ligamentoso a través del arco de movimiento que puede provocar por un lado contractura y por otro inestabilidad. El aumento de la presión del aparato extensor se produce por el desplazamiento anterior del componente femoral, resección insuficiente de las facetas rotulianas y una malrotación de los componentes femoral o tibial. Un sobredimensionamiento del componente femoral compromete la articulación femoropatelar y disminuye el espacio en flexión, con la consecuente limitación de la flexión de la rodilla. Un componente tibial demasiado alto lleva a un aumento de la tensión en flexión y extensión, con lo que habrá la tendencia a producir una contractura en flexión con limitación de la misma. Una pendiente anterior del corte tibial lleva a limitar la flexión.

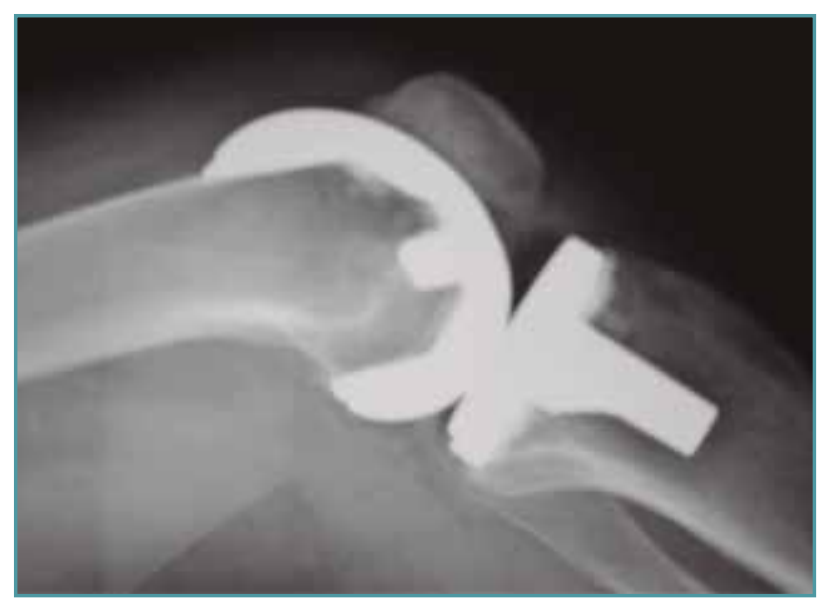

Figura 1. Rodilla rígida tras artroplastia por "patela baja".

En cuanto al balance ligamentoso, un aumento de la tensión de los ligamentos laterales o del ligamento cruzado posterior (LCP) también conduce a la rigidez articular.

Las complicaciones postoperatorias más frecuentes de una rodilla rígida son la infección, la fractura periprotésica, la descementación de los componentes, la distrofia simpático-refleja y las osificaciones heterotópicas ${ }^{(5,13)}$.

\section{Tratamiento}

Para realizar un correcto tratamiento es necesario identificar la etiología y, solo en caso de no tener un diagnóstico de certeza, vamos a reconocer la artrofibrosis como causa de la rigidez, como un proceso de metaplasia del fibrocartílago con incremento de la fibrosis intersticial(13,4) (Figuras 2 y 3). La historia clínica ayuda a pensar en los problemas de cicatrización, la presencia de infección superficial en el postoperatorio inmediato, los traumatismos y en la aparición de una rigidez $z^{(15,16)}$. Un cuadro que aparece en el postoperatorio inmediato lleva a pensar que se trata de un error quirúrgico o una rehabilitación inadecuada, mientras que en el caso de una rigidez de aparición tardía, tras un periodo de movilidad satisfactoria, nos encaminará a un proceso de infección latente o descementación.

En la exploración clínica buscaremos signos flogóticos, inestabilidad o compatibles con una distrofia simpático-refleja. En los controles ra- 


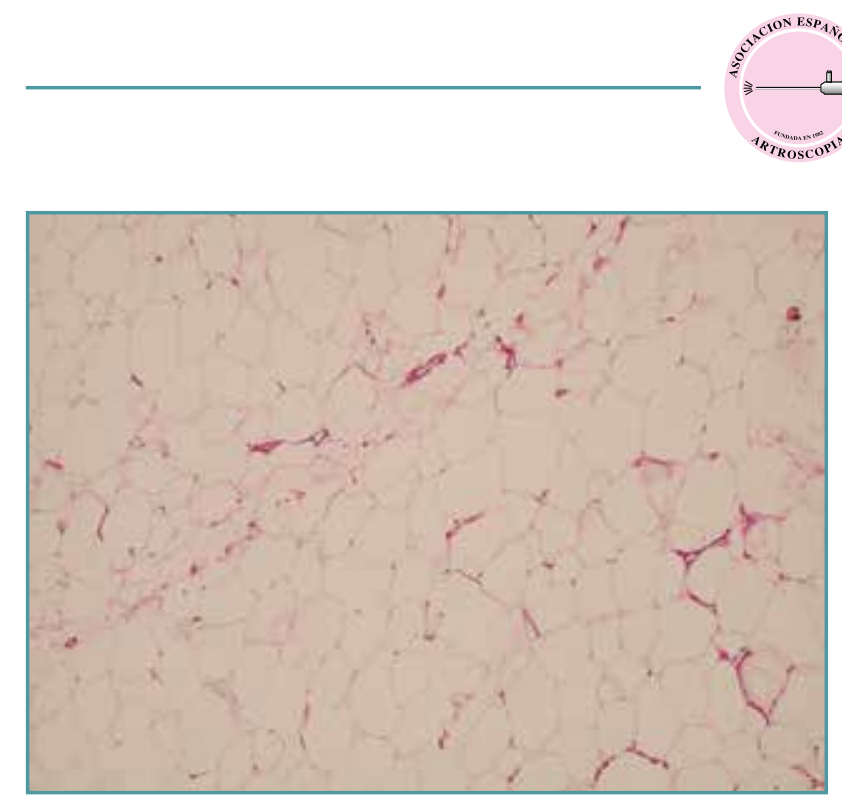

Figura 2. Patrón intersticial normal de la grasa de Hoffa.

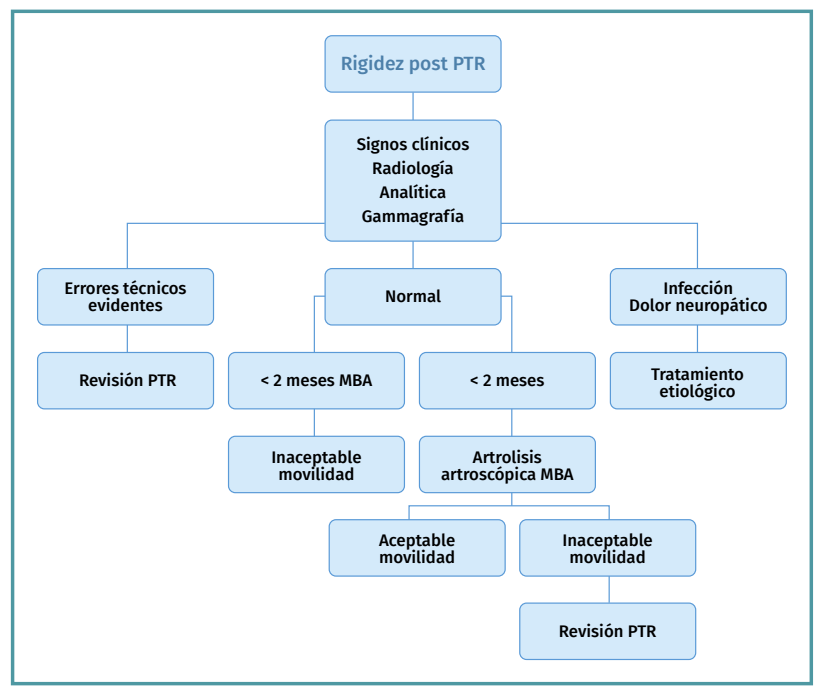

Figura 4. Algoritmo de tratamiento en rigidez de rodilla después de una artroplastia.

diográficos descartaremos problemas de medida y colocación de los componentes protésicos, descementación de los mismos o un corte inadecuado de la interlínea articular. Los estudios isotópicos permiten diagnosticar la presencia de una descementación protésica, infección o un cuadro de distrofia simpático-refleja. Los parámetros biológicos son los que descartan un proceso inflamatorio o séptico (velocidad de sedimentación, proteína $C$ reactiva, hemograma y estudio del líquido articular).

En los casos de rigideces precoces, el tratamiento incluye una rehabilitación agresiva con

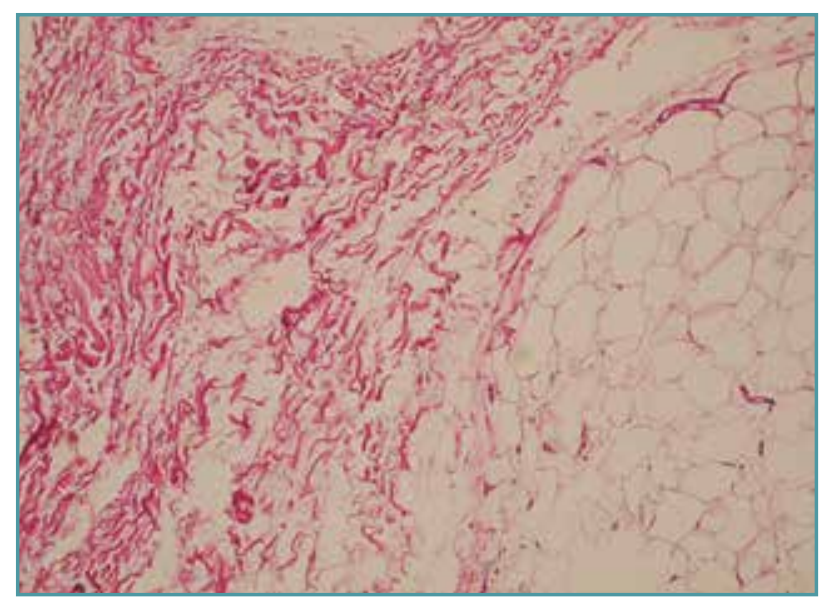

Figura 3. Patrón de fibrosis intersticial del paquete adiposo de Hoffa en rodilla rígida.

una analgesia adecuada y suficiente, con ayuda de férulas dinámicas y, si es preciso, antes de las primeras 8 semanas. Puede ser de ayuda una manipulación bajo anestesia cuando no se demuestran errores de malposicionamiento de la prótesis ${ }^{(5,10)}$; es discutible la práctica de una artroscopia pues existen autores que tienen buenos resultados con la artrolisis artroscópica con liberación del LCP ${ }^{(17,18)}$, mientras otros ${ }^{(19)}$ refieren que solo una tercera parte mejoran a largo plazo. Sin embargo, los resultados son mejores, mejora la movilidad articular, con la artrolisis artroscópica ${ }^{(20-28)}$ que con la manipulación bajo anestesia (Figura 4).

\section{Técnica quirúrgica}

Con el paciente anestesiado y en completa relajación, se comprueba la situación real de la rigidez y el arco de movimiento. Se utilizan los portales habituales, inferolateral (IL), inferomedial (IM) y superolateral (SL), procediendo desde este último a la introducción de suero salino preferiblemente con sistema de bomba a presión para distender lo máximo posible la cápsula articular. Algunos autores ${ }^{(26-28)}$ recomiendan el uso de cánulas de plástico en los portales para evitar lesionar las superficies del componente femoral o del inserto de polietileno. Se deben practicar tantos portales accesorios como sean necesarios en función de una correcta visibilidad y facilitar la instrumentación: anterior (Gillquist), posteromedial (PM) o posterolateral (PL). 


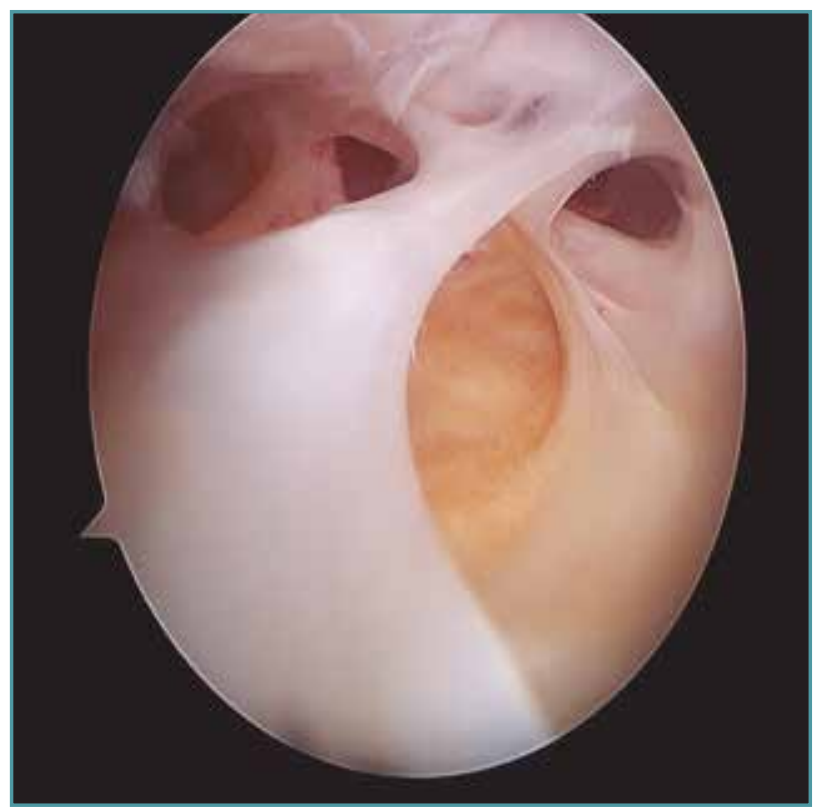

Figura 5. Fibrosis en el receso medial en la rodilla rígida.

El desbridamiento se inicia, en primer lugar, por el espacio intercondíleo. Desde el portal IL se introduce la óptica y desde el IM la instrumentación, procediendo a la extirpación de la fibrosis en el intercóndilo. Se reseca a motor todo el tejido periligamentario e incluso el LCP desde su origen femoral, en casos de ser portador de un implante con retención del LCP, así como la fibrosis en cara retropatelar y grasa de Hoffa ${ }^{(26-28)}$.

Siguiendo por los recesos lateral y medial (Figura 5) se llega a la zona suprapatelar, cuya fibrosis es la primera causa de limitación de la flexión (Figura 6). En este espacio hay que ser muy meticuloso para resecar con motor (Figura 7) y radiofrecuencia las adherencias, los nódulos, cíclopes (Figura 8), la fascia profunda del cuádriceps, con esqueletización de la cara anterior de fémur si es necesario, y liberar el alerón externo e interno, en función de la rótula: si está lateralizada (liberación solo lateral) o bien centrada (liberación lateral y medial), para facilitar el recorrido rotuliano. La limpieza de este espacio es fundamental para recuperar el máximo grado de flexión.

Con la misma instrumentación se liberan de adherencias ambos recesos laterales, restos meniscales y se debe despegar la cápsula de las paredes lateral y medial de los cóndilos. A veces, para llegar a visualizar estas estructuras es ne-

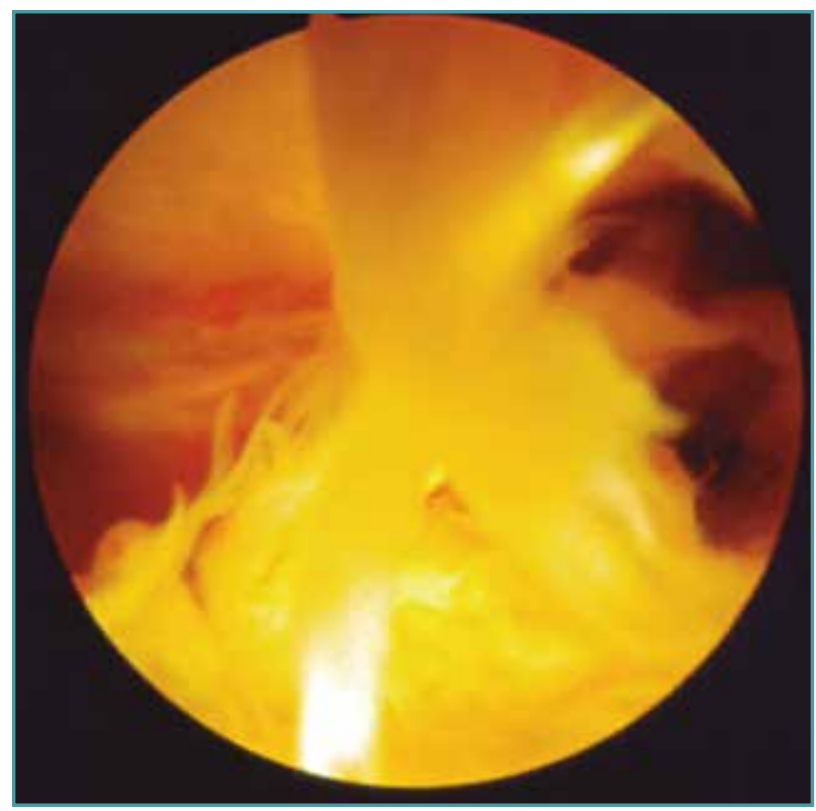

Figura 6. Bandas fibrosas articulares con disfunción patelofemoral y dolor anterior en prótesis de rodilla.

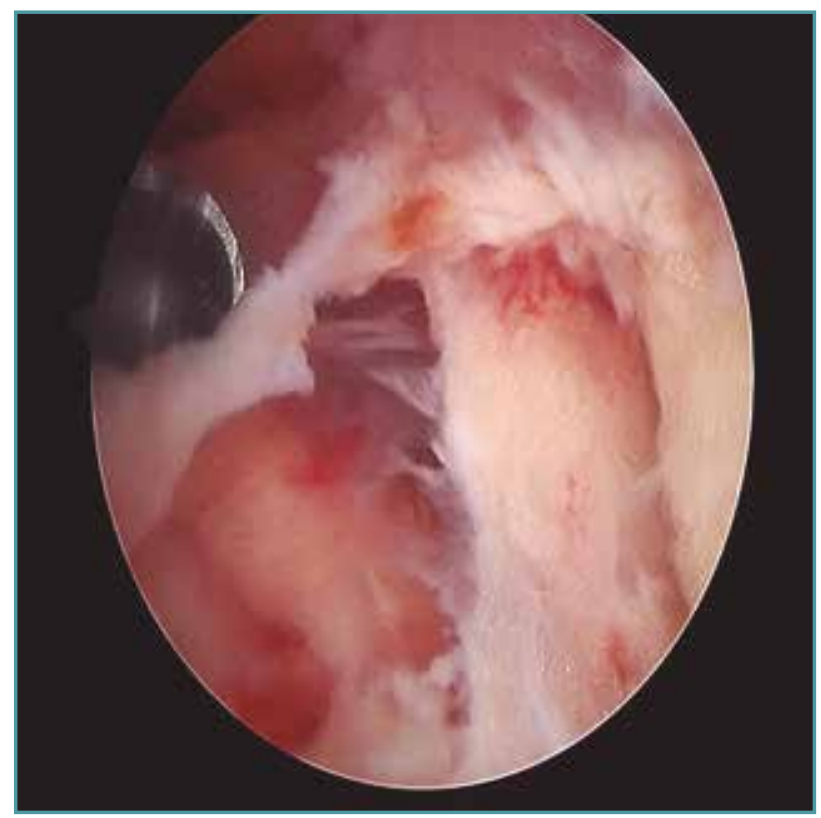

Figura 7. Resección a motor de las bandas fibrosas en el espacio supracondíleo.

cesario utilizar la óptica de $70^{\circ}$. Una vez se ha conseguido movilizar la rótula, volvemos a limpiar el espacio pretibial de los restos de grasa de Hoffa y adherencias del tendón rotuliano ${ }^{(27,28)}$. En este momento, se procede a revisar la estabilidad 


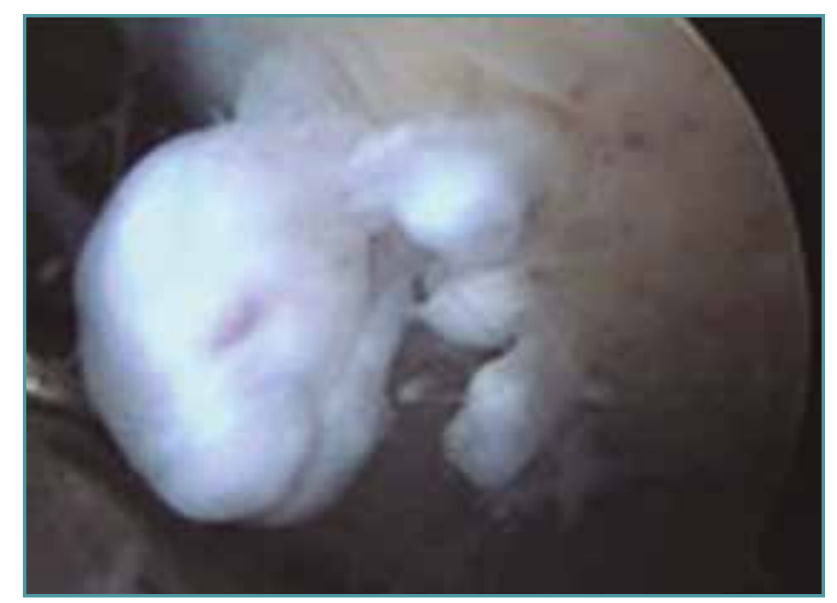

Figura 8. Nódulo fibroso entre polo superior de rótula y tendón cuadricipital. Resalte y dolor en prótesis con estabilización posterior.

de los componentes protésicos así como la presencia de cuerpos libres, restos de cemento que protruyen en el espacio metal-hueso y se pueden tomar biopsias si hay sospecha de infección. Se puede comprobar posibles atrapamientos de tejidos interpuestos entre los componentes protésicos o roces del ligamento poplíteo con el inserto y de la fascia lata con la bandeja tibial externa.

Si la contractura en flexión persiste, debemos acceder al compartimento posterior mediante los portales PM y PL desde visión anterior, efectuar la limpieza de tejido fibroso y practicar una liberación de la cápsula posterior, del tejido fibroso que puede rozar con la cara posterior del polietileno y desinsertar la cápsula de la cara posterior del fémur.

Durante todo el procedimiento y en diferentes momentos de las liberaciones, se puede intentar de forma suave la manipulación bajo anestesia, siempre con especial cuidado de evitar el arrancamiento de la tuberosidad tibial ${ }^{(27,28)}$.

En el postoperatorio inmediato se debe utilizar un sistema de movimiento pasivo continuo, así como fisioterapia intensiva para mantener la movilidad conseguida durante el procedimiento quirúrgico.

La artrolisis abierta implica un desbridamiento radical con liberación del LCP, reequilibrando las partes blandas, con sección del alerón externo $y$, si es preciso, relajando el aparato extensor, mediante una cuadricepsplastia o una osteotomía de la tuberosidad tibial anterior $(5,6,10,12)$. Cuando se

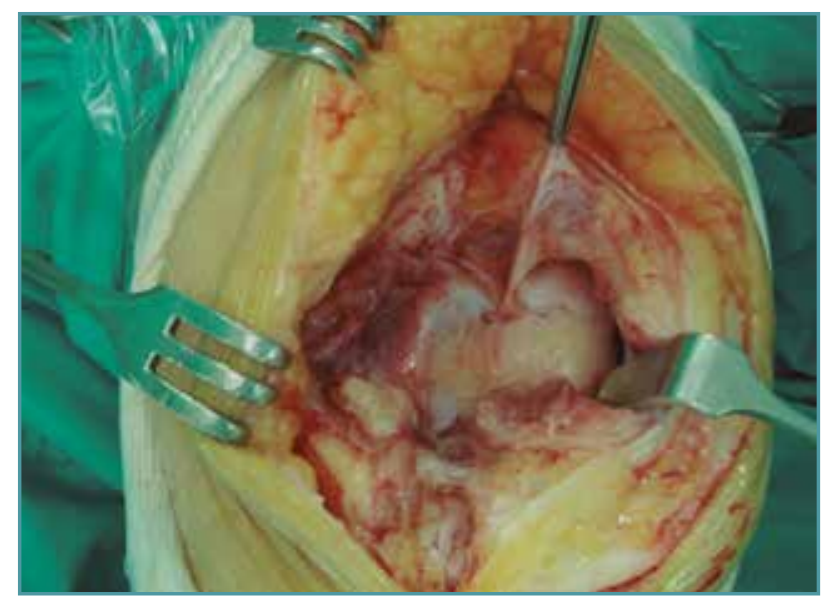

Figura 9. Adherencias en fondo de saco cuadricipital.

objetiva un error en la mala implantación de los componentes, estará además indicado realizar su revisión (Figura 9).

Antes de realizar una revisión quirúrgica debemos considerar si el paciente presenta una limitación funcional o un dolor suficiente que la justifique. La intervención estará contraindicada si existe un motivo extrínseco de la rigidez que es irresoluble (alteraciones de la cadera, alteraciones neurológicas, contracturas musculares, alteraciones inflamatorias...) $)^{(6)}$. El conocimiento del motivo de la rigidez ayuda a tener unos mejores resultados; sin embargo, es infrecuente obtener el rango de movimiento "normal" tras el tratamiento de la misma ${ }^{(5,6,10)}$.

\section{Bibliografía}

1. Aglietti P, Windsor RE, Buzzi R, Insall JN. Arthroplasty for the stiff or ankylosed knee. J Arthroplasty. 1989;4:1-5.

2. Scranton PE Jr. Management of knee pain and stiffness after total knee arthroplasty. I Arthroplasty. 2001;16:428-35.

3. Christensen CP, Crawford JJ, Olin MD, Vail TP. Revision of stiff total knee arthroplasty. J Arthroplasty. 2002;17:409-15.

4. Nicholls DW, Dorr LD. Revision surgery for stiff total knee arthroplasty. J Arthroplasty. 1990;5(Suppl):73-7.

5. Scuderi GR. The stiff total knee arthroplasty. J Arthroplasty. 2005;20:23-6. 
6. Nelson CL, Kim J, Lotke PA. Stiffness after total knee arthroplasty. J Bone Joint Surg (Am). 2005;87-A(Suppl.):264-70.

7. Harden RN, Bruehl S, Stanos S, Brander V, Chung OY, Saltz $S$, et al. Prospective examination of pain-related and psychological predictors of CRPS-like phenomena following total knee arthroplasty: a preliminary study. Pain. 2003;106:393-400.

8. Fox JL, Poss R. The role of manipulation following total knee replacement. J Bone Joint Surg (Am). 1981;63A:357-62.

9. Markel DC, Luessenhop CP, Windsor RE, Sculco TA. Arthroscopic treatment of peripatella fibrosis after total knee arthroplasty. J Arthroplasty. 1996;11:293-7.

10. Cuckler JM. The Stiff Knee: Evaluation and management. Orthopedics. 2002;25:969.

11. Laubenthal KN, Smidt GL, Kettlekamp DB. A quantitative analysis of knee motion during activities of daily living. Phys Ther. 1972;52:34-43.

12. Kim J, Nelson CL, Lotke PA. Response bias: effect on outcomes evaluation by mail surveys after total knee arthroplasty. J Bone Joint Surg (Am). 2004;86-A:15-21.

13. Kurosaka M, Yoshiya S, Mizuno K, Yamamoto T. Maximizing flexion after total knee arthroplasty: the need and the pitfalls. J Arthoplasty. 2002;17(suppl 1):59-62.

14. Ries MD, Badalamente M. Arthrofibrosis after total knee arthroplasty. Clin Orthop Relat Res. 2000; 380:177-83.

15. Daluga D, Lombardi AV Jr, Mallory TH, Vaughn BK. Knee manipulation following total knee arthroplasty: analysis of prognostic variables. J Arthroplasty. 1991;6:119-28.

16. Mauerhan DR, Mokris JG, Ly A, Kiebzak GM. Relationship berween length of stay and manipulation rate after total knee arthroplasty. J Arthroplasty. 1998;13:896-900.

17. Dennis DA. Problems after Knee arthroplasty. The stiff total knee arthroplasty: causes and cures. Orthopedics. 2001;24:901-2.
18. Ritter MA, Harty LD, Davis KE, Dvis KE, Meding JB, Berend ME. Predicting range of motion after total knee arthroplasty. J Bone Joint Surg (Am). 2003;85-A:1278-85.

19. Williams RJ III, Westrich GH, Siegel J, Windsor RE. Arthroscopic release of posterior cruciate ligament for stiff total knee arthroplasty. Clin Orthop Rel Res. 1996;331:185-91.

20. Diduch DR, Scuderi GR, Scott WN, Insall JN, Kelly MA. The efficacy of arthroscopy following total knee replacement. Arthroscopy. 1997;13:166-71.

21. Fitzsimmons SE, Vazquez EA, Bronson MJ. How to treat the stiff total knee arthroplasty? A systematic review. Clin Orthop Relat Res. 2010;468:1096-106.

22. Jerosch J, Aldawoudy AM. Arthroscopic treatment of patients with moderate arthrofibrosis after total knee replacement. Knee Surg Sports Traumatol Arthrosc. 2007;15:71-7.

23. Schwarzkopf R, William A, Deering RM, Fitz W. Arthroscopic lysis of adhesions for stiff total knee arthroplasty. Orthopedics. 2013;36:1544-8.

24. Williams RJ III, Westrich GH, Siegel J, Windsor RE. Arthroscopic release of the posterior cruciate ligament for stiff total knee arthroplasty. Clin Orthop Relat Res. 1996;331:185-91.

25. Hegazy AM, Elsoufy MA. Arthroscopic arthrolysis for arthrofibrosis of the knee after total knee replacement. HSS J 2011;7:130-3.

26. Arbuthnot JE, Brink RB. Arthroscopic arthrolysis for the treatment of stiffness after total knee replacement gives moderate improvements in range of motion and functional knee scores. Knee Surg Sports Traumatol Arthrosc. 2010;18:346-51.

27. Enad JG. Arthroscopic lysis of adhesions for the stiff total knee arthroplasty. Arthrosc Tech. 2014:3:611-4.

28. Tjoumakaris FB, Tucker BS, Post Z, Pepe MD, Orozco F, Ong AC. Arthroscopic lysis of adhesions for the stiff total knee: results after failed manipulation. Orthopedics. 2014;37482-7. 